\title{
Long-Lasting Input-Specific Experience- Dependent Changes of Hippocampus Synaptic Function Measured in the Anesthetized Rat
}

\author{
Eliott R. J. Levy, ${ }^{{ }^{\wedge}}$ Kally C. O’Reilly, ${ }^{1}{ }^{\wedge}$ and ${ }^{(1)}$ André A. Fenton ${ }^{1,2,3}$
}

https://doi.org/10.1523/ENEURO.0506-18.2019

${ }^{1}$ Center for Neural Science, New York University, New York, NY 10003, ${ }^{2}$ Neuroscience Institute at the New York University Langone Medical Center, New York, NY 10016, and ${ }^{3}$ Department of Physiology, the Robert F. Furchgott Center for Neural and Behavioral Science, SUNY Downstate Medical Center, Brooklyn, NY 11203

\begin{abstract}
How experience causes long-lasting changes in the brain is a central question in neuroscience. The common view is that synaptic function is altered by experience to change brain circuit functions that underlie conditioned behavior. We examined hippocampus synaptic circuit function in vivo, in three groups of animals, to assess the impact of experience on hippocampus function in rats. The "conditioned" group acquired a shock-conditioned place response during a cognitively-challenging, hippocampus synaptic plasticity-dependent task. The no-shock group had similar exposure to the environmental conditions but no conditioning. The home-cage group was experimentally naive. After the one-week retention test, under anesthesia, we stimulated the perforant path inputs to CA1, which terminate in stratum lacunosum moleculare $(\mathrm{s} / \mathrm{m})$, and to the dentate gyrus (DG), which terminate in the molecular layer. We find synaptic compartment specific changes that differ amongst the groups. The evoked field EPSP (fEPSP) and pre-spike field response are enhanced only at the DG input layer and only in conditioned animals. The DG responses, measured by the population spiking activity and post-spike responses, are enhanced in both the conditioned and no-shock groups compared to home-cage animals. These changes are pathway specific because no differences are observed in sIm of CA1. These findings demonstrate long-term, experience-dependent, pathway-specific alterations to synaptic circuit function of the hippocampus.
\end{abstract}

Key words: active place avoidance; conditioning; experience; hippocampus; memory; synaptic circuit function

\section{Significance Statement}

We investigated whether a hippocampus-dependent place avoidance memory causes long-lasting changes of hippocampus circuit function, as is commonly assumed. Immediately after testing one-week memory retention in rats, under anesthesia, the entorhinal cortex (EC) projection to dentate gyrus (DG) is strengthened, as estimated by the synaptic response to stimulation as well as the amplitude of the population action potential response. These changes are pathway specific because no differences are observed in the stratum lacunosum moleculare $(\mathrm{slm})$ of CA1 where the EC projection also terminates. These findings of experience-dependent, pathway-specific alterations to synaptic circuit function in hippocampus are consistent with theories that posit that memory formation causes persistent alterations of neural circuit function.

Received December 24, 2018; accepted August 10, 2019; First published August 21, 2019

The authors declare no competing financial interests.
Author contributions: E.R.J.L., K.C.O., and A.A.F. designed research; E.R.J.L. and K.C.O. performed research; E.R.J.L. and K.C.O. analyzed data; E.R.J.L., K.C.O., and A.A.F. wrote the paper. 


\section{Introduction}

How experience alters the brain to enable conditioned responses is an open question. The current view is that changes to synaptic circuit function underlie conditioned behavior. Changes to synaptic function have been difficult to identify and have focused on long-term potentiation (LTP) immediately after conditioning a behavior (Whitlock et al., 2006), although changes in synaptic function have also been observed during (Fernández-Lamo et al., 2018) or for one or more days after conditioning (Gruart et al., 2006; Park et al., 2015; Pavlowsky et al., 2017).

The two-frame active place avoidance task conditions a place response that depends on synaptic modifications in the dorsal hippocampus, specifically $\mathrm{PKM} \zeta$-dependent and CaMKII-dependent LTP (Pastalkova et al., 2006; Tsokas et al., 2016; Hsieh et al., 2017; Rossetti et al., 2017). These studies have not, however, identified if the modifications to synaptic circuit function are universal or pathway-specific. In mice, following two-frame active place avoidance conditioning, robust changes were observed specifically in the CA3-CA1 synaptic response, but not in the entorhinal cortex (EC)-CA1 response, in ex vivo hippocampus slice recordings (Pavlowsky et al., 2017). In anesthetized mice, place avoidance training enhanced the excitability of granule cells in the dentate gyrus (DG) in response to EC stimulation (Park et al., 2015). Here, we extend these findings to the rat. Immediately after retraining one week after task acquisition, under anesthesia, we find training-induced changes in hippocampus function that are specific to the DG but not the CA1 targets of the EC inputs.

\section{Materials and Methods}

All methods complied with Public Health and Service Policy on Humane Care and Use of Laboratory Animals and were approved by New York University Animal Welfare Committee, which follow National Institutes of Health guidelines.

\section{Animals}

Twenty-two male Long-Evans (Charles River) rats arrived at the New York University animal facilities and were given at least a week to acclimate. The rats had free access to food and water and were single housed.

\section{Two-frame active place avoidance}

Sixteen rats were used for the behavioral experiments. Rats were handled $\sim 5 \mathrm{~min} / \mathrm{d}$ for $5 \mathrm{~d}$ before behavioral training. The training took place on an 81-cm diameter circular disk-shaped rotating arena with transparent walls

This work was supported by National Institutes of Health Grants $\mathrm{R} 01 \mathrm{MH} 099128$ and $\mathrm{R} 01 \mathrm{MH} 115304$.

${ }^{\wedge}$ E.R.J.L. and K.C.O. contributed equally to this work.

Correspondence should be addressed to André A. Fenton at afenton@nyu.edu.

https://doi.org/10.1523/ENEURO.0506-18.2019

Copyright (C) 2019 Levy et al.

This is an open-access article distributed under the terms of the Creative Commons Attribution 4.0 International license, which permits unrestricted use, distribution and reproduction in any medium provided that the original work is properly attributed.
(Bio-Signal Group). The arena could be stationary or rotate at $1 \mathrm{rpm}$. The position of a rat on the arena was monitored at 30 frames per second from an overhead camera using video-tracking software (Tracker, BioSignal Group). The software could track the rat's position in the room as well as on the rotating arena and could deliver a mild constant current foot shock $(0.3$ $\mathrm{mA}, 60 \mathrm{~Hz} 500 \mathrm{~ms}$ ) whenever the rat was detected in a $60^{\circ}$ sector that was designated the shock zone. Although the arena rotated, the shock zone was stationary and was defined by stationary landmarks that were fixed in the room.

The rats were given two 10-min pre-training trials to habituate to the stationary arena. During the two training days (sessions 1 and 2), the rats were given eight 10-min trials per day on the rotating arena. Rats in the conditioned group $(n=8)$ received shocks in the shock zone and rats in the no-shock group $(n=8)$ experienced the same physical conditions, except they were never shocked. The time between trials was $\sim 10 \mathrm{~min}$. One week later, the rats were all returned to the rotating arena for 10 min to test memory retention. The shock was on in the shock zone for rats in the conditioned group. Immediately after the one-week experience the rats were anesthetized and prepared for assessing in vivo hippocampus evoked responses. The home-cage $(n=6)$ group consisted of rats that were never exposed to the behavioral room and were not handled (Fig. 1A).

\section{Behavioral assessment}

Behavioral end-point measures were computed from the position timeseries using TrackAnalysis (Bio-Signal Group). We evaluated the distance walked on the arena, the number of entrances made into the shock zone, the time spent in each quadrant, and the time to enter the shock zone for the first time. The distance walked on the arena assesses locomotion. The shock zone entrances and the time in each quadrant estimate place avoidance and preference. The time to first enter the shock zone estimates avoidance memory across sessions.

\section{In vivo hippocampus-evoked responses}

Surgical protocol

Immediately after the one-week retention trial, rats were anesthetized with $1.2 \mathrm{~g} / \mathrm{kg}$ urethane (intraperitoneal) and placed in a stereotaxic frame. A 16-site linear-array silicon electrode with $30-\mu \mathrm{m}$ diameter recording contacts and 100- $\mu \mathrm{m}$ spacing (Neuronexus; p/n: A $1 \times 16$ - 5mm-100703) was placed in the dorsal hippocampus to span the somatodendritic axis of dorsal CA1 and DG (Fig. 1B). A six-wire stimulating electrode bundle was placed in the ipsilateral perforant path. The stimulating electrodes were made by twisting six nichrome wires $(75 \mu \mathrm{m})$ together. The wires were cut at an angle to allow the six sites to span $1 \mathrm{~mm}$. The following coordinates were used to target the perforant path fibers: relative to bregma, anteroposterior $(-7.6 \mathrm{~mm})$, mediolateral $(4.1$ $\mathrm{mm})$, and dorsoventral $(2.5-3.0 \mathrm{~mm})$. The two-wire bipolar combination that evoked the largest response was selected for the stimulation experiments. A constant current stimulus isolation unit (WPI; model: 
A
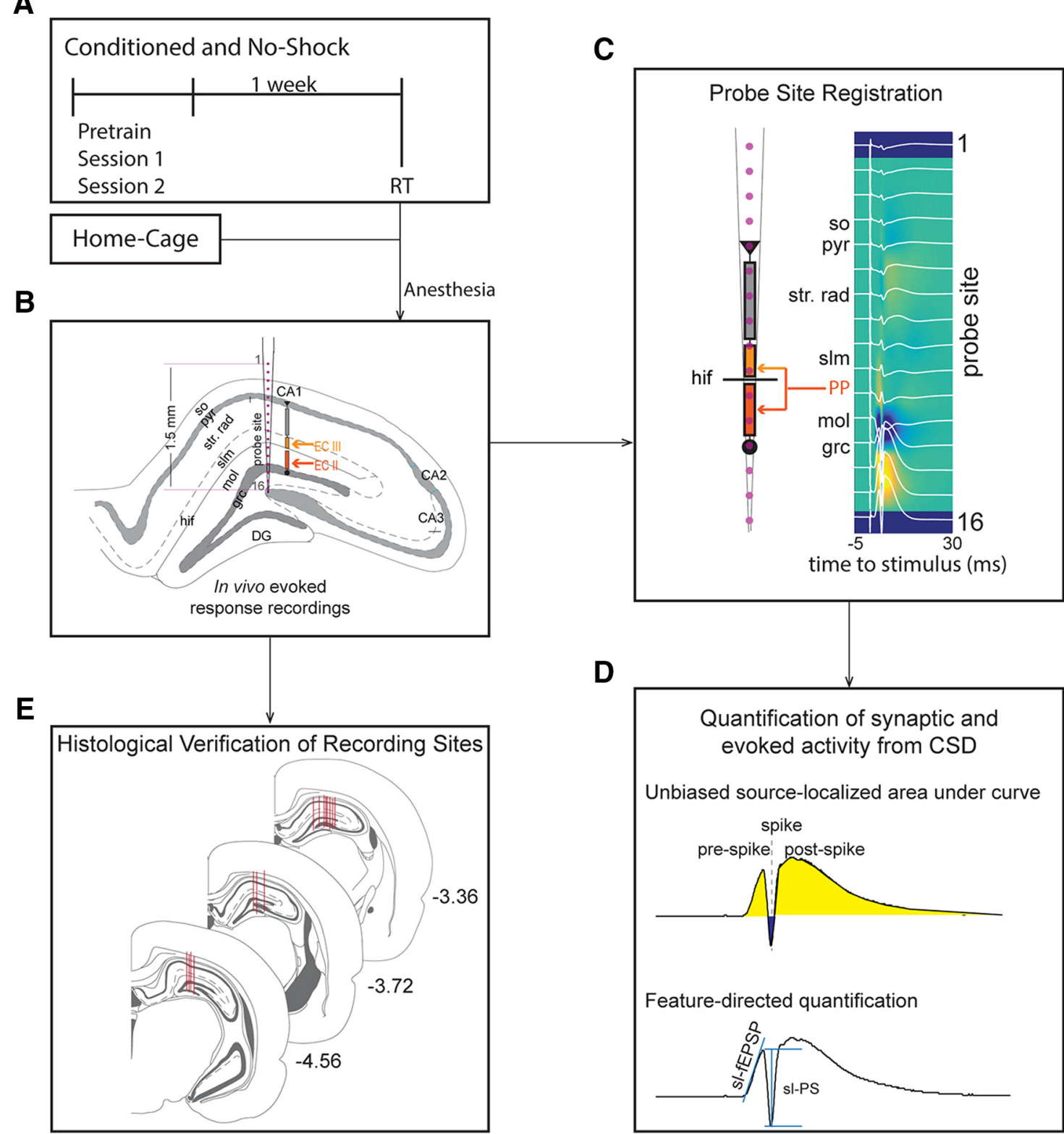

Figure 1. Evoked response paradigm. $\boldsymbol{A}$, Immediately after the retention trial, the conditioned and no-shock rats were anesthetized and underwent surgery for the evoked potential experiments. Home-cage rats were never handled before the evoked response recordings. B, A 16-site linear electrode array was placed in the dorsal hippocampus, spanning the somatodendritic axis of CA1 and the DG. A bundle of stimulating electrodes was placed in the perforant path to stimulate EC inputs. $\boldsymbol{C}$, The recording probes were localized by performing CSD analysis, which identifies sinks and sources of the stereotypical response to perforant path stimulation at each layer. $\mathrm{CA1}$ : so = stratum oriens, $\mathrm{pyr}=$ pyramidal cell layer, strat. rad. = stratum radiatum, $\mathrm{DG}$ : $\mathrm{mol}=\mathrm{molecular}$ layer, grc $=$ granule cell layer, hif = hillar fissure. $\boldsymbol{D}$, Quantification of the synaptic and evoked activity from the CSD was performed either by calculating the AUCs for sinks and sources before or after the PS activity and was performed for each layer of CA1 and DG. The other approach quantified the fEPSP of the molecular and granule cell layers and population spiking activity of the granule cell layer. $\boldsymbol{E}$, Recording sites were verified histologically. Red lines indicate positions of the recording probes for the experiments performed. Anteroposterior coordinates relative to bregma are indicated next to the coronal sections.

A365RC) was used to deliver individual $150-\mu$ s stimulus pulses across the electrode pair.

The response to stimulation was recorded using Axona USBdacq software and the Axona hardware that was optimized for recording evoked potential responses. An attenuating resistor (either 18 or $47 \mathrm{k} \Omega$ ) was connected to the Axona input to maximize the effective number of bits
(16) used for digitization. The signals were low-pass filtered $<5 \mathrm{kHz}$ and digitized at $48 \mathrm{kHz}$.

\section{Localization of the recording electrodes}

Current source density (CSD) analysis was performed for source localization, minimizing volume conducted signals in the analyzed data. The CSD is computed from the 
second spatial derivative of the voltage along the recording depth:

$$
\operatorname{csD}(z)=-\frac{V(z-\Delta z)+V(z+\Delta z)-2 * V(z)}{\Delta z^{2}}
$$

where CSD (z) is the CSD at depth $z, V(z)$ is the Voltage at depth $z$ and $\triangle z=100 \mu \mathrm{m}$ is the distance between adjacent recording sites. The CSD is calculated in units of $\mathrm{mV} / \mu \mathrm{m}^{2}$ and can be multiplied by a conductivity constant $\sigma$ to measure it in units of $A / \mu \mathrm{m}^{3}$. By convention, CSD is computed as the negative of the second spatial derivative with negative CSD indicating an extracellular current sink and positive CSD an extracellular current source. Because it is isotropic (Hrabetová, 2005), we assume that the conductivity is homogeneous within the hippocampus as it was shown that variations in the conductivity had little effect on hippocampus CSD estimates (Holsheimer, 1987). Similarly, the conductivity was assumed to be similar across the groups.

The pattern of sinks and sources in each animal was used to localize the electrode recording sites to each somatodendritic location for an individual rat (Fig. 1C) as previously described (Brankack et al., 1993; Wu and Leung, 2003). Briefly, the DG granule cell layer was identified by the largest source in the lower channels that also included a sink, which was the population spike (PS) activity. The molecular layer was identified as the largest sink one to two channels above the granule cell layer. The stratum lacunosum moleculare ( $\mathrm{s} / \mathrm{m}$ ) was identified by two channels: the large sources above the molecular layer and an early latency sink in the one to two channels just above the largest source. Stratum radiatum was identified as a late latency sink and the pyramidal layer was identified as a late source above stratum radiatum. Stratum oriens was identified as the sink above the pyramidal layer. Based on the sinks and sources from one animal from the conditioned group and two animals from the no-shock group, the recording and/or stimulation electrodes did not appear to be properly placed and these three animals were excluded from the physiology analyses. Thus, for the physiology data, the final group numbers were conditioned $n=7$, no-shock $n=6$, home-cage $n=6$.

\section{Analysis of the responses evoked by stimulation}

All analyses were performed offline using custom MAT$L A B$ software. We performed all estimates of the evoked responses on the CSD traces to minimize the impact of volume conduction. Accordingly, we refer to these evoked responses as source-localized (sl). Input-output (I-O) curves were generated by measuring the evoked responses to stimulus intensities ranging from 100 to 1000 $\mu \mathrm{A}$ in $100-\mu \mathrm{A}$ steps. At each stimulus intensity, four voltage responses were recorded and the CSD computed for each response. All measurements were performed on each of the four responses and then averaged. Spike times were estimated at the DG-granule cell layer for each rat.

To visualize the responses along the somatodendritic axis, we aligned all recordings to the DG granule cell layer compartment and computed the group average CSD re- sponse to $800-\mu \mathrm{A}$ stimulation for each rat. We chose to compute the average at $800 \mu \mathrm{A}$ because that was the highest stimulation intensity delivered to all animals. The CSDs obtained at $800 \mu \mathrm{A}$ were then averaged for each group to visualize the group-specific sinks and sources. The data were plotted with interpolation across adjacent electrodes using the shading interp MATLAB function.

Unbiased quantification: area under the curve (AUC) We computed the AUC of the source localized (sI-) evoked response as an unbiased estimate of the response since it includes both direct and polysynaptic responses as well as spiking. The source-localized AUC (sl-AUC) was measured before and after population spiking at each compartment and separately for sources and sinks (Fig. 1D). Analysis of the individual sources and sinks within the CSD profile allowed us to quantify specific components of the response, such as the post-spike activity, that is otherwise difficult to identify and measure as it is comprised of excitatory and inhibitory responses. We note that interpreting an (inward) current sink or (outward) current source depends on knowing the functional anatomy of the relevant sites because active sinks and sources are associated with passive return sources and sink currents, respectively.

Feature-directed quantification The evoked response contributions to the field EPSP (fEPSP) and to the PS are well-characterized and commonly used to estimate the responses of DG to entorhinal stimulation. Feature directed analysis was performed on the evoked CSD traces to measure the source-localized PS (sI-PS) and fEPSP (sl-fEPSP; Fig. 1D). The sl-PS was measured in the granule cell layer of the DG and two measures of the sl-fEPSP were made: the positive slope in the granule cell layer and the negative slope in the molecular layer. The time window for estimating sl-fEPSP was $1.5 \mathrm{~ms}$ after the stimulus was delivered until $1 \mathrm{~ms}$ before population spiking activity. This time restriction was chosen to prevent the inclusion of (variable) dendritic spiking activity (Herreras, 1990).

\section{Boltzmann function fits}

We examined fEPSP and PS (E-S) coupling in the DG by fitting a Boltzmann function to the data using the following equation for the sl-PS as a function of the sl-fEPSP:

$$
s l-P S=\frac{s l-P S_{\max }}{1+\exp \left(\frac{s l-f E P S P_{50}-s l-f E P S P}{S}\right)}
$$

Where $s /-P S_{\max }$ is the maximum sl-PS, $s /-f E P S P_{50}$ is the $s l-f E P S P$ associated with the $50 \%$ sl-PS max response, and $S$ is the slope (the slope being steeper as $S$ decreases). The equation was fit for each animal and the $s /-P S_{\text {max }}$, sl-fEPSP $P_{50}$ and slopes were compared among the groups.

\section{Paired-pulse inhibition}

Stimulation to assess paired-pulse inhibition was performed at $65 \%$ of the intensity required to elicit the maximal sl-PS response. Stimulus pairs were delivered at increasing interstimulus intervals between the first and 
second pulses $(5,10,20,40,80,160,320$, and $640 \mathrm{~ms})$. We allowed $30 \mathrm{~s}$ between each pair of stimuli. At each interstimulus interval, four responses were recorded and CSD analysis was performed to attenuate effects of volume conduction. The responses were measured and the ratio of the second to first response was used to estimate the amount of inhibition on the second stimulation due to the initial stimulation. These ratios were averaged across the four recordings.

\section{Verification of stimulation and recording sites}

At the end of the recordings, the rats were transcardially perfused with $1 \times$ PBS followed by $10 \%$ formalin. The brains were extracted and stored in formalin overnight and stored in $30 \%$ sucrose in $1 \times$ PBS until they were cut on a cryostat $(40 \mu \mathrm{m})$ and thaw mounted onto gelatincoated slides. The sections were dried overnight at room temperature and then Nissl stained. The slides were scanned at 10X with an Olympus VS120 microscope and the images were subsequently examined for electrode tracks to verify the stimulation and recording locations (Fig. 1E).

\section{Statistical analysis}

Statistical analysis was performed in JMP 14 (SAS). Behavioral data from all the animals were included in the analysis (conditioned: $n=8$; no-shock $n=8$ ). Multivariate ANOVA (MANOVA) was conducted for each behavioral phase (pretraining, training, retention) separately. For the pretraining and training phases, we conducted MANOVA analyses with the trials used as a repeated measure; Hotelling's trace correction was used when sphericity was violated. Additionally, when relevant, two-group comparisons were made using Student's $t$ tests, with degrees of freedom adjusted for unequal variances. Electrophysiological data were assessed by ANOVA to identify statistically significant group, stimulation intensity, and group $\times$ stimulation intensity differences between conditioned, noshock, and home-cage rats. Stimulation intensity was treated as a continuous variable. Tukey's honest significant difference (HSD) post hoc tests evaluated pair-wise differences. The effect size $\eta^{2}$ is given when a nonsignificant statistical trend is observed. Statistical significance was set at $p<0.05$.

\section{Results}

\section{Conditioned behavior}

The arena is stationary during the pretraining trials, allowing the assessment of locomotor behavior, similar to that done in an open field test. Because the rats were randomly assigned to the conditioned and no-shock groups, we did not expect to see group differences in locomotor activity on the stationary arena. Indeed, before training, the conditioned and no-shock control groups are identical in their exploratory activity (Fig. 2B). The average time spent in each location for all rats in each group (Fig. $2 B$, heat maps), with example traces of a rat from each group (Fig. $2 B$, gray traces), indicates that during pretraining, rats do not avoid going into the region that will eventually be designated as the shock zone. However, to investigate biases for locations on the arena, we analyzed the proportion of time spent in each quadrant. There is a quadrant effect $\left(F_{(3,56)}=3.12, p=0.03\right)$ because rats show a preference to spend time in at least one quadrant, but the group interactions are not significant, indicating no influence of group (group $\times$ quadrant: $F_{(3,56)}=0.41, p$ $=0.74$; group $\times$ quadrant $\times$ trial: $\left.F_{(3,56)}=0.16, p=0.92\right)$, and neither is the trial $\times$ quadrant interaction significant, indicating steady state preferences $\left(F_{(3,56)}=0.82, p=\right.$ 0.49). Although it appears from the heat maps (Fig. $2 B$ ) that the animals are spending more time in the quadrant opposite the eventual shock zone, post hoc analysis on the quadrants indicates no preference for this specific quadrant. Furthermore, the two groups are indistinguishable in their behaviors during this initial exploratory period (Fig. $2 C-E$ ) in the total distance walked on the stationary arena (group: $F_{(1,14)}=0.04, p=0.85$; trial: $F_{(1,14)}=10.52$, $p=0.006$; group $\times$ trial: $\left.F_{(1,14)}=0.78, p=0.39\right)$, the number of times they enter the eventual shock zone (group: $F_{(1,14)}=0.23, p=0.64$; trial: $F_{(1,14)}=2,27, p=$ 0.15 ; group $\times$ trial: $\left.F_{(1,14)}=0.95, p=0.34\right)$ and the latency to first enter the eventual shock zone (group: $F_{(1,14)}$ $=8 \times 10^{-4}, p=0.98$; trial: $F_{(1,14)}=4.77, p=0.05$; group $\times$ trial: $\left.F_{(1,14)}=0.55, p=0.47\right)$.

Conditioning the rats to avoid the location of the shock during the training trials results in multiple behavioral differences. Conditioned rats learn to avoid the location of shock on the rotating arena in the two-frame active place avoidance task whereas rats exposed to the same environment without shock (no-shock rats) do not express a conditioned place response (Fig. 2B). During training, an effect of quadrant is observed $\left(F_{(3,112)}=10.53, p=10^{-6}\right)$ as well as a group $\times$ quadrant interaction $\left(F_{(3,112)}=26.34\right.$, $p=10^{-13}$ ) and a group $\times$ trial $\times$ quadrant effect $\left(F_{(21,215.08)}=1.7, p=0.032\right)$, but no other interaction (trial $\times$ quadrant effect: $F_{(21,215.08)}=1.54, p=0.067$; day $\times$ quadrant effect: $F_{(3,112)}=0.20, p=0.90$; group $\times$ day $\times$ quadrant effect: $F_{(3,112)}=0.65, p=0.58$; trial $\times$ day $\times$ quadrant effect: $F_{(21,215.08)}=1.37, p=0.13$; group $\times$ trial $\times$ day $\times$ quadrant effect: $\left.F_{(21,215.08)}=1.32, p=0.17\right)$. Post hoc tests done separately for each group during training show an effect of sector for both groups but with a preference for the opposite sectors: Trained animals spend the least time in the shock quadrant while noshock animals spend the most time there. Both groups of rats appear to have an increase in distance walked early in session 1 compared to what is observed in pretraining, which may be due to the novelty of being on a rotating arena that was previously stationary. As seen in the example trajectory and the group-averaged time spent in each location on the arena in the last training trial of session 2 (Fig. 2B), the conditioned rats learn to avoid the shock zone. The conditioned rats walk $18 \%$ less than the no-shock rats during training (Fig. $2 \mathrm{C}$ ), which is because of the no-shock rats' increased locomotion, and due to the restriction of the conditioned rats walking in only three of the four quadrants (distance walked; group: $F_{(1,28)}=$ 9.76, $p=4.12 \times 10^{-3}$; day: $F_{(1,28)}=0.82, p=0.37$; trial: $F_{(7,22)}=1.57, p=0.20$; group $\times$ day: $F_{(1,28)}=0.67, p=$ 0.42 ; group $\times$ trial: $F_{(7,22)}=1.4, p=0.26$; day $\times$ trial: $F_{(7,22)}=1.26, p=0.31 ;$ group $\times$ day $\times$ trial: $F_{(7,22)}=0.60$, 
A

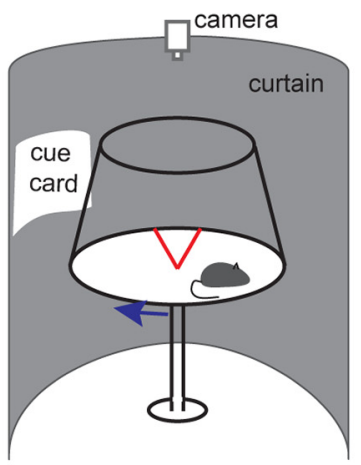

B

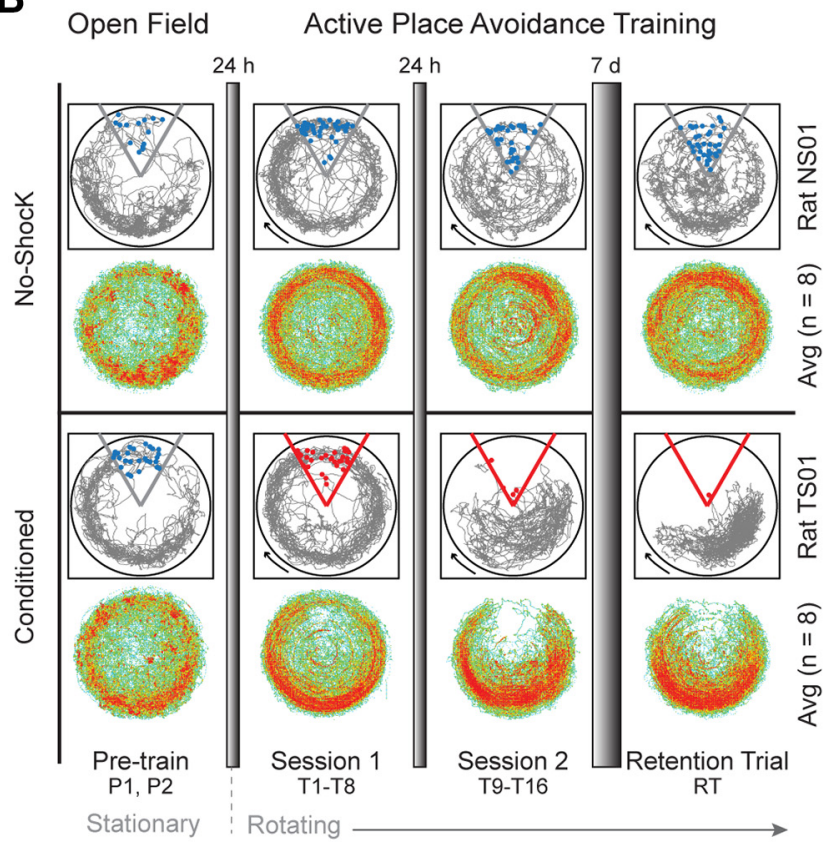

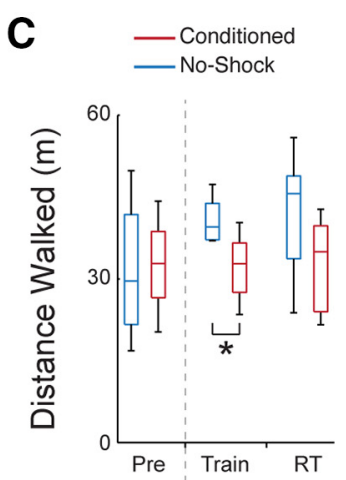

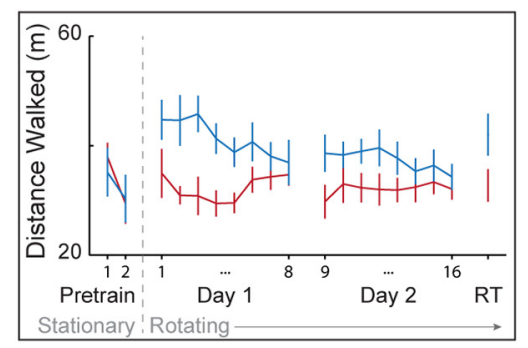

D
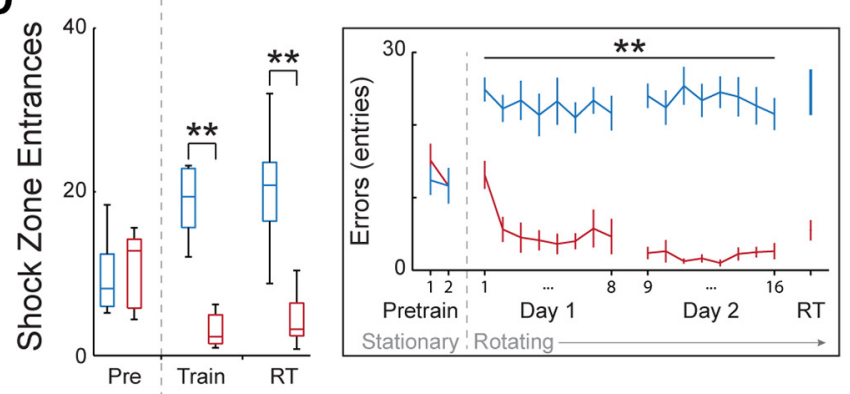

E

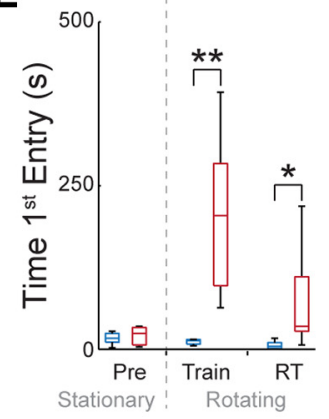

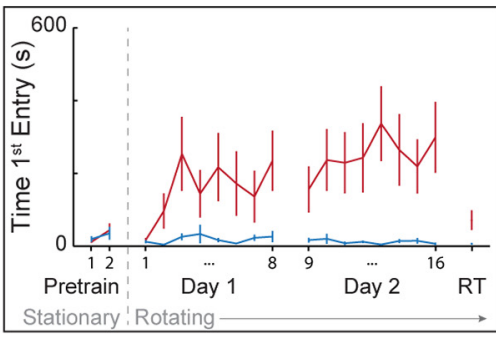

Figure 2. Memory-related behavior is different between conditioned and no-shock rats. $\boldsymbol{A}$, Rats were placed on a rotating arena and conditioned to avoid a mild foot shock within a $60^{\circ}$ sector that was stationary within the room. The no-shock group was exposed to the same conditions, but without foot shock. $\boldsymbol{B}$, The rats were given two pretraining trials on a stationary arena without shock. The next day the rats started $2 \mathrm{~d}$ of conditioning (sessions 1 and 2). Each day a conditioning session consisted of eight training trials. One week after the end of conditioning, the rats were given a retention trial to test memory. For the conditioned group, the retention trial was conducted with the shock on. All trials were $10 \mathrm{~min}$ with at least $10 \mathrm{~min}$ intertrial intervals. Example paths (in gray) of one no-shock rat and one conditioned rat are shown over the course of training. The red dots represent where the animal received shocks and blue dots indicate where the animal would have received shock if the shock had been present. The heat maps show average time spent for all rats in each $0.3 \times 0.3-\mathrm{cm}$ region for each group. Red represents an average dwell of $>0.017 \mathrm{~s}$. By the end of the training period, the conditioned rats avoid the $60^{\circ}$ sector (bottom) while the no-shock rats do not exhibit a place bias. $\boldsymbol{C}-\boldsymbol{E}$, Box plots of behavioral measures for the pre-training session, the training sessions (trials 1-16), and the retention trial show there are differences between no-shock and conditioned rats. Asterisks in the box plots (C-E, left) indicate a main effect of group, while asterisks in the line graph ( $C-E$, right) indicate group differences. no-shock, $n=8$; conditioned, $n=8$. Plots to the right of the boxplots represent mean \pm SEM on a trial-by-trial basis; $* p<0.05, * * p<0.01$.

$p=0.75)$. The conditioned group quickly reduces the number of entries into the shock zone while the no-shock rats continue to enter the shock zone location throughout the entire training period (shock zone entrances; group: $F_{(1,28)}=171.12, p=10^{-13}$; day: $F_{(1,28)}=0.97, p=0.33$; trial: $F_{(7,22)}=1.50, p=0.22$; group $\times$ day: $F_{(1,28)}=2.04$, $p=0.16$; group $\times$ trial: $F_{(7,22)}=2.55, p=0.044$; day $\times$ trial: $F_{(7,22)}=0.7, p=0.67$; group $\times$ day $\times$ trial: $F_{(7,22)}=$ $0.65, p=0.71$; post hoc Student's $t$ tests to evaluate group differences at each trial are all significant at $p<$
0.05; Fig. 2D). Conditioned rats also show avoidance memory by increasing their latency to enter the shock zone over the course of conditioning (time to first entry; group: $F_{(1,28)}=29.57, p=10^{-6}$; day: $F_{(1,28)}=1.43, p=$ 0.24; trial: $F_{(7,22)}=0.84, p=0.56$; group $\times$ day: $F_{(1,28)}=$ $1.91, p=0.18$; group $\times$ trial: $F_{(7,22)}=1.60, p=0.19$; day $\times$ trial: $F_{(7,22)}=0.36, p=0.92$; group $\times$ day $\times$ trial: $F_{(7,22)}$ $=0.14, p=0.99$; Fig. $2 E$ ).

The impact of the training experience on behavioral measures persists for at least one week following the end 
of conditioning. During the retention trial, an effect of quadrant $\left(F_{(3,56)}=4.49, p=6.80 \times 10^{-3}\right)$ and a group $\times$ quadrant interaction is still observed $\left(F_{(3,56)}=9.36, p=\right.$ $10^{-5}$ ). Again, post hoc tests done separately on each group during the retention trial show that conditioned rats spend the least amount of time in the shock quadrant $\left(F_{(3,28)}=9.42, p=1.82 \times 10^{-4}\right.$ with Tukey's post hoc indicating preference for the quadrant opposite to the shock quadrant), which is also seen in the reduced number of entrances into the shock zone compared to noshock rats $\left(t_{(9.81)}=5.95, p=1.52 \times 10^{-4}\right)$. During the retention trial, the no-shock rats show no preference for any of the quadrants $\left(F_{(3,28)}=0.97, p=0.42\right)$ and there is no significant group difference in the distance walked $\left(t_{(13,18)}=1.91, p=0.078\right)$. In addition to displaying a preference for avoiding the shock quadrant, the conditioned rats have an increased latency to enter the shock quadrant $\left(t_{(7.09)}=2.42, p=0.046\right)$, another indicator that the conditioned rats have a one-week-old place avoidance memory.

\section{Evoked potential results}

We examined synaptic function in the dorsal hippocampus under anesthesia immediately following the retention trial. As is seen by the overlapping traces of the voltage and the CSD response waveforms (Fig. 3A), performing CSD analysis reduces the influence of volume conduction on the waveforms and is therefore a better localized estimate of the evoked activity at the different somatodendritic compartments of CA1 and DG. We registered the data to the somatodendritic locations and then calculated group average CSD traces (Fig. 3B), which appear different, although we did not further analyze the spatial profile of the CSD.

All estimates of evoked activity were performed on the CSD-corrected responses and are referred to as sourcelocalized. To evaluate the stimulation responses, we chose to take two separate approaches, a feature-directed quantification and an unbiased evaluation of the evoked responses. The first approach is to quantify specific features of the evoked response that are traditionally used to estimate the synaptic and spiking components, the slope of the evoked sl-fEPSP response and the amplitude of the sl-PS, respectively. The second approach evaluates the evoked activity localized to a single electrode at a specific location along the somatodendritic axis. Without relying on assumptions about the waveform of the response, we measured the AUC of the CSD trace at this site. The activity following spiking is particularly difficult to quantify using traditional methods but can be captured in a straightforward manner by measuring the AUC of the response that occurs after the PS. Whether this activity corresponds to active transmembrane currents or passive return currents cannot be determined from the CSD alone and requires knowledge of the functional anatomy for a complete interpretation, and we therefore analyzed the CSD source and sink components separately. In contrast, the activity preceding the PS standardly estimated by the slope of the fEPSP can also be estimated by measuring the AUC before the PS, which quantifies the entire response instead of the response at a specific time point; these two estimates of the synaptic response at the molecular layer are correlated in our dataset $\left(r=0.87, p=10^{-57}\right)$.

To get an initial sense of where training-induced changes in the evoked response might occur, we quantified the source-localized activity before and after the PS activity by measuring the sl-AUC in response to $800-\mu \mathrm{A}$ stimulation, which is the maximum stimulation intensity that was recorded for all animals (Fig. 3C). Because we wanted to separately quantify the activity before and after the sl-PS, we first determined if the sl-PS times differed among the groups. While the sl-PS times do differ across the three groups $\left(F_{(2.89)}=3.72, p=0.03\right)$ as well as by stimulation intensity $\left(F_{(1,89)}=9.68, p=2.5 \times 10^{-3}\right)$, there is no interaction $\left(F_{(2,89)}=0.01, p=0.99\right)$ and no post hoc group differences. While the pre-spike activity at the molecular layer is not different between groups $\left(F_{(2,16)}=2.05\right.$, $p=0.16, \eta^{2}=0.20$; Fig. $3 C$, left), the post-spike sink at the molecular layer is significantly different among the groups $\left(F_{(2,16)}=3.65, p=0.0496\right)$. The difference between the conditioned and home-cage groups was confirmed by post hoc tests, but neither group is different from the no-shock group (Fig. $3 \mathrm{C}$, right). Although the pre-spike molecular layer sink represents the perforant path input, it is uncertain to what extent this difference in the post-spike molecular layer sink reflects a traininginduced difference in either an active transmembrane current or a passive return current.

We evaluated the I-O curves of pre- and post-spiking responses (sl-AUC) at the molecular layer (Fig. 3D), both of which show a significant effect of group due to greater statistical sensitivity of repeating the measurements for different stimulus intensities. The pre-spike response plateaus at $\sim 300-\mu \mathrm{A}$ stimulation for all groups and is greater for the conditioned group (group: $F_{(2,180)}=17.38, p=$ $10^{-7}$; stimulation intensity: $F_{(1,180)}=2.93, p=0.09$; interaction: $F_{(2,180)}=0.39, p=0.68$; with post hoc differences between the conditioned group and the other groups; Fig. $3 D$, top). After the spike, the response increases with stimulation intensity and is different between the groups such that conditioned $>$ no-shock $>$ home-cage (group: $F_{(2,180)}=23.92, p=10^{-10}$; stimulation intensity: $F_{(1,180)}=$ 99.25, $p=10^{-19}$; interaction: $F_{(2,180)}=6.04, p=0.003$; with post hoc differences between all groups; Fig. $3 D$, bottom).

Traditionally, the early slope of the fEPSP is used to estimate the synaptic response and the PS amplitude is used to estimate synchronous neuronal discharge. We estimated these values from the CSD-corrected responses. The conditioned group sl-fEPSP at the molecular layer is larger than that of the no-shock and homecage rats (group: $F_{(2,180)}=13.65, p=10^{-6}$; stimulation intensity: $F_{(1,180)}=48.33, p=6.41 \times 10^{-11}$; interaction: $F_{(2,180)}=2.30, p=0.10$; conditioned $>$ no-shock $=$ home-cage; Fig. 3E).

Although the overall CSD responses (Fig. $3 C$ ) do not appear different among the groups at the granule cell layer, there is a group effect on both the sl-fEPSP slope (group: $F_{(2,180)}=3.79, p=0.02$; stimulation intensity: 
A

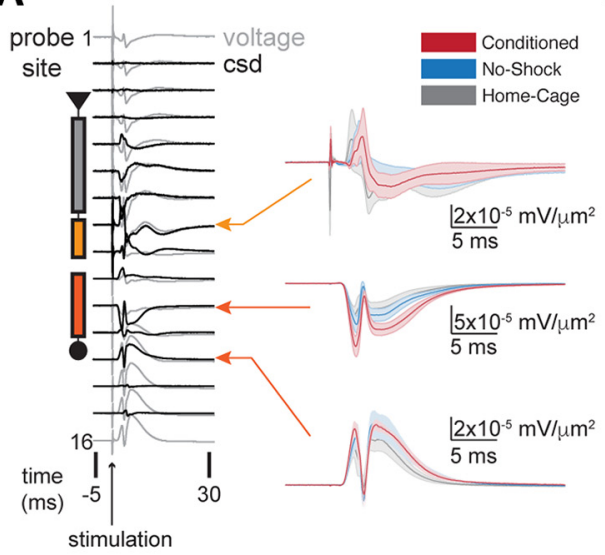

C

Pre-Spike

sl-AUC $\left(\times 10^{-7} \mathrm{mV} \cdot \mathrm{s} / \mu \mathrm{m}^{2}\right)$ at $800 \mu \mathrm{A}$

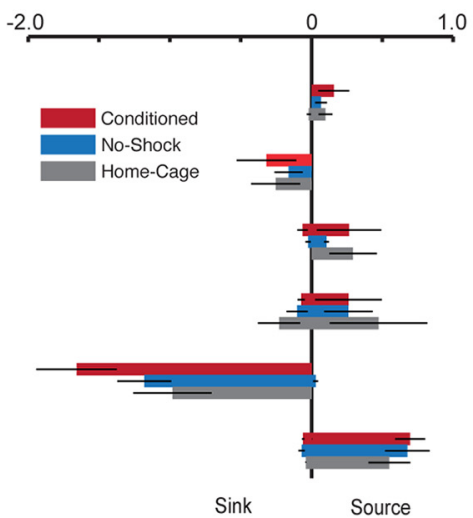

E

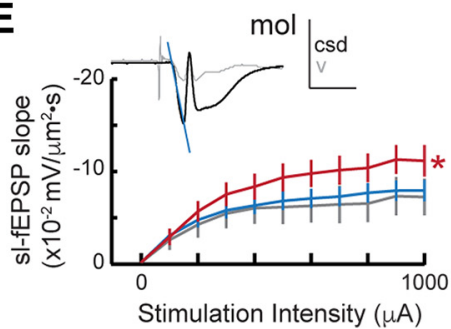

B

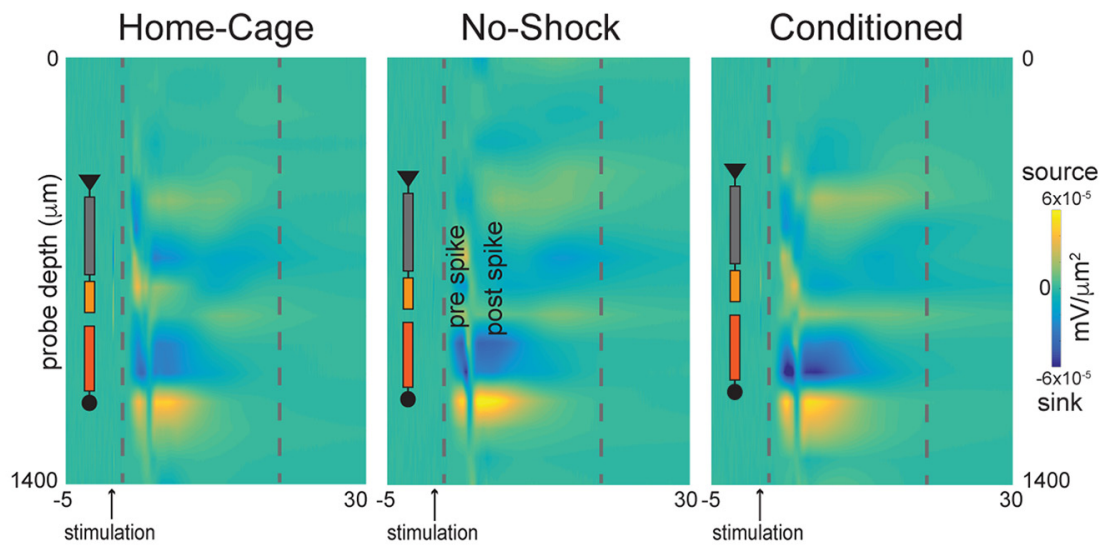

D

Post-Spike $\mathrm{sl}-\mathrm{AUC}\left(\times 10^{-7} \mathrm{mV} \cdot \mathrm{s} / \mu \mathrm{m}^{2}\right)$ at $800 \mu \mathrm{A}$

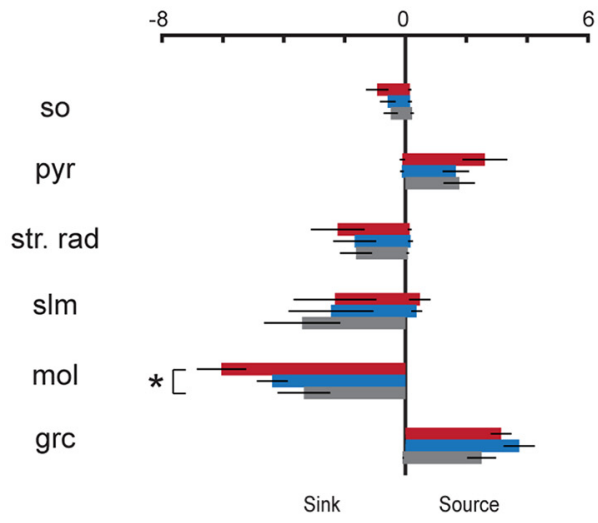

$\overline{\bar{O}}$

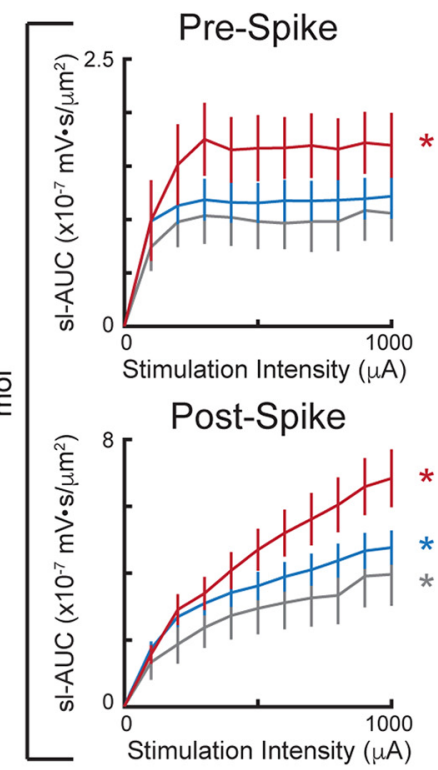

G

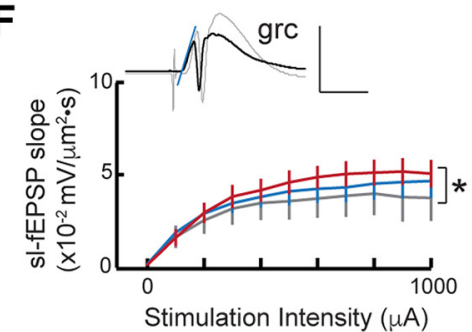

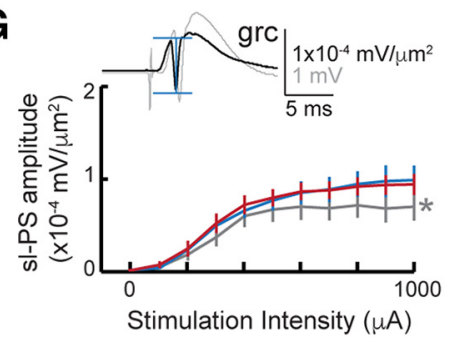

Figure 3. Behavior-induced alterations to DG circuit function assessed by response to perforant path stimulation. $\boldsymbol{A}$, The CSD (black traces) was computed from the recorded voltage traces (gray) to remove volume conducted signals. The sites determined to be located in slm, molecular layer and granule cell compartments (orange arrows) were used for further evaluation. The average \pm SEM (shading) sl-CSD are shown. B, Average CSD heatmaps were generated for each group. Cooler colors are sinks (inward current) and warmer colors are sources (outward current). The sink in the molecular layer appears to increase with experience and cognitive demand. $\boldsymbol{C}$, The AUC of the pre-spike response (1.5 ms after the stimulation was delivered until the spike occurred) and the post-spike response (from the spiking activity until $20 \mathrm{~ms}$ after stimulation) were quantified at the maximum stimulation given to all animals (800 $\mu \mathrm{A})$ for each layer of the somatodendritic axis of the dorsal hippocampus. The pre-spike sink at the molecular layer is not changed by experience whereas the post-spike sink in the molecular layer is significantly altered by experience. CA1: so = stratum oriens, pyr = pyramidal cell layer, strat. rad. = stratum radiatum, DG: mol = molecular layer, grc = granule cell layer. $D$, top, The I-O curve that characterizes the pre-spike sink, synaptic population response to the full range of stimulus intensities, is significantly increased in conditioned rats compared to both no-shock and home-cage groups. Bottom, The post-spike response to increasing stimulation intensity is significantly increased in conditioned rats compared to the no-shock response, which is also significantly increased compared to home-cage rats. $\boldsymbol{E}$, The sl-fEPSP characterizing the population synaptic response at the molecular layer is significantly increased by conditioning, confirming the findings based on the sl-AUC. $\boldsymbol{F}$, The sl-fEPSP at the granule cell layer is significantly 
continued

different between conditioned and home-cage groups. G. The PS response at the granule cell layer is significantly increased by experience. Example CSD waveforms are plotted in $\boldsymbol{E}-\boldsymbol{G}$ in black for illustration with schematization of the measurement corresponding to the figure. The voltage trace is underlaid in gray. Home-cage, $n=6$; no-shock, $n=6$; conditioned, $n=7$. Data are mean \pm SEM. $* p<0.05$. Colored asterisks indicate which group is different from the other two.

$F_{(1,180)}=35.23, p=10^{-8}$; interaction: $F_{(2,180)}=0.79, p=$ 0.46 ; conditioned $>$ no-shock $=$ home-cage; Fig. $3 F)$ and the sl-PS amplitude (group: $F_{(2,180)}=5.92, p=0.003$; stimulation intensity: $F_{(1,180)}=160.76, p=10^{-27}$; interaction: $F_{(2,180)}=1.64, p=0.20$; conditioned $=$ no-shock $>$ home-cage; Fig. 3G). None of these parameters measured in the physiology correlate with the behavior, in particular with the time to first enter the shock zone on the retention trial (data not shown). These differences are also not accompanied by differences in E-S coupling [molecular layer sl-fEPSP to granule cell layer sl-PS (Fig. $4 A, B$ ): sl-PS $\max : F_{(2,16)}=1.24, p=0.32, \eta^{2}=0.13$; sl-fEPSP ${ }_{50}$ : $F_{(2,16)}=1.00, p=0.39, \eta^{2}=0.11$; slope: $F_{(2,16)}=1.82, p$ $=0.19, \eta^{2}=0.19$; granule cell layer sl-fEPSP to granule cell layer sl-PS (Fig. $4 C, D$ ): sl-PS $\max : F_{(2,16)}=0.86, p=$ $0.44, \eta^{2}=0.10$; sl-fEPSP $50: F_{(2,16)}=0.36, p=0.70, \eta^{2}=$ 0.04 ; slope: $\left.F_{(2.16)}=1.18, p=0.33, \eta^{2}=0.13\right]$. Although the slopes of the E-S coupling appear different (Fig. 4B,D,
A
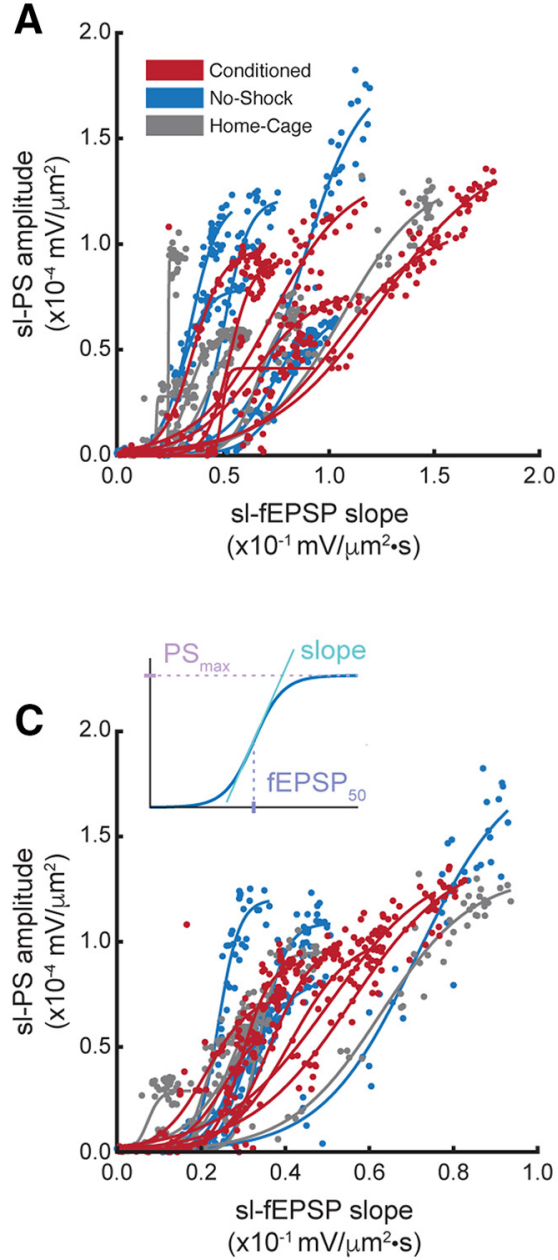

sl-fEPSP ${ }_{\text {mol }}$ to $s /-P S_{\text {grc }}$ coupling

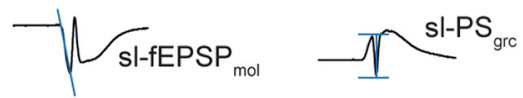

B
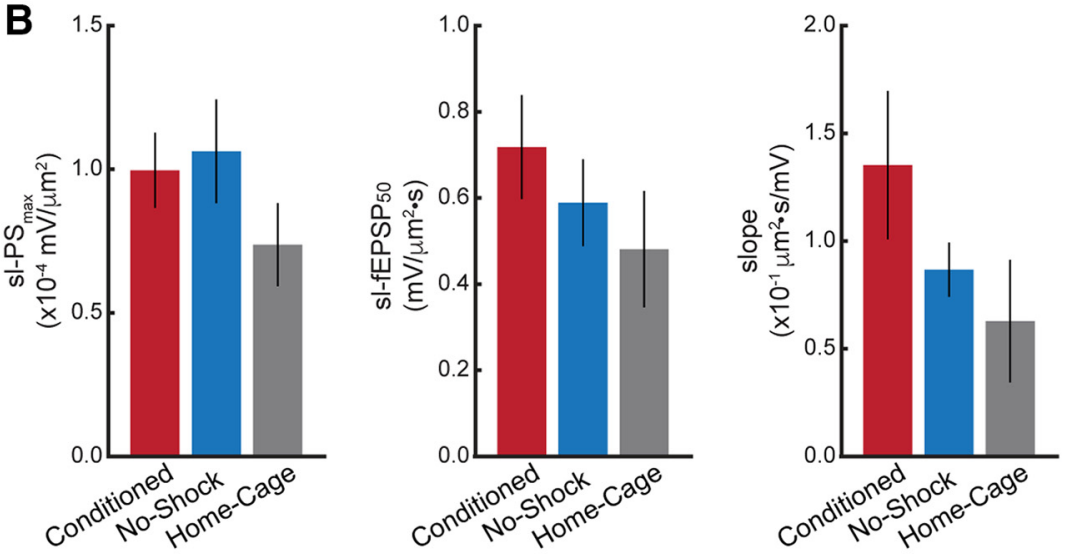

Sl-fEPSP $P_{\text {grc }}$ to $S I-P S_{\text {grc }}$ coupling
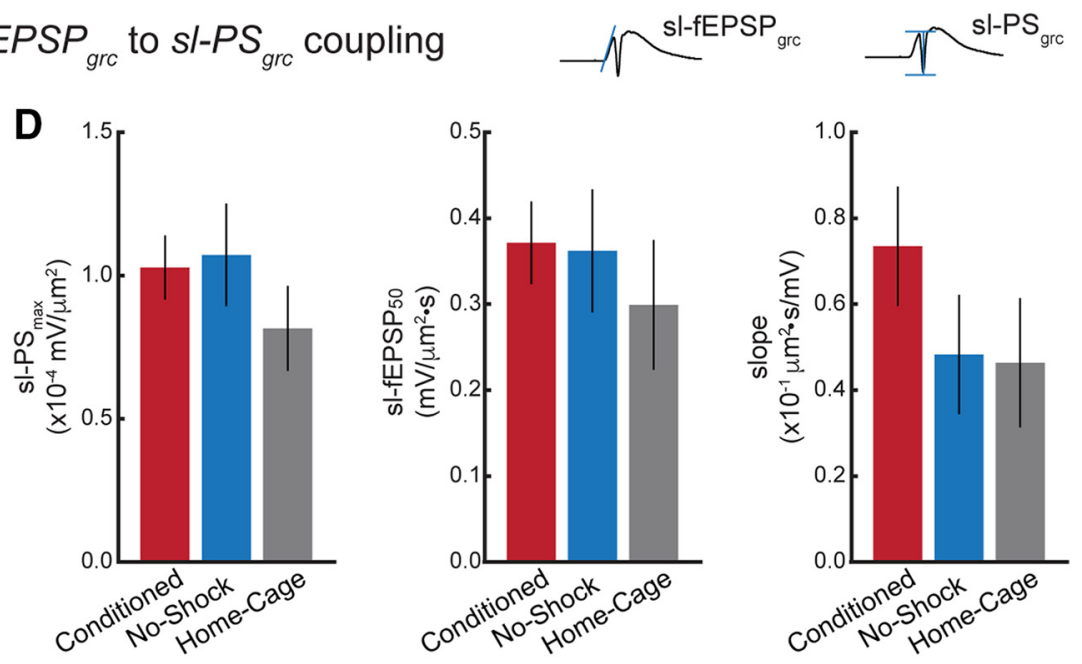

Figure 4. Coupling between the field synaptic potentials and population spiking is not different between groups. $\boldsymbol{A}$, The sl-fEPSP measured at the molecular layer is plotted against the sl-PS measured at the granule cell layer for each stimulation response, with intensity varying from 100 to $1000 \mu \mathrm{A}$. The Boltzmann fit to each animal's E-S coupling is overlaid. $\boldsymbol{B}$, Average parameters of the

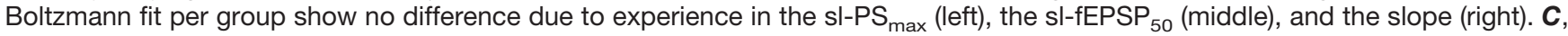
The sl-fEPSP measured at the granule cell layer is plotted against the sl-PS measured at the granule cell layer. $\boldsymbol{D}$, Average parameters

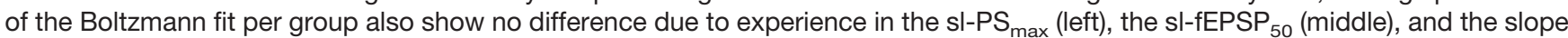
(right). The inset in $\boldsymbol{C}$ schematizes the role of the parameters of the Boltzmann fit. The example CSD waveforms in $\boldsymbol{B}, \boldsymbol{D}$ are plotted for illustration. 
right panel), significance is not reached, likely due to the small effect sizes. Indeed, a power analysis with power set to 0.80 indicates three to four times larger sample sizes are needed to detect group differences in the E-S coupling. Finally, the differences in sl-fEPSP and sl-PS cannot not be easily attributed to changes in feedback inhibition, as there is no difference in paired-pulse inhibition measured as the ratio sl-PS2/sl-PS1 (group: $F_{(2,113)}=$ $0.13, p=0.88$; ISI: $F_{(1,113)}=2.73, p=0.10$; interaction: $F_{(2,113)}=0.49, p=0.61$; data not shown). Nonetheless, other estimates of altered inhibition merit assessing (Ruediger et al., 2011).

\section{Discussion}

\section{Summary}

Here, we show that one week after two-frame active place avoidance conditioning, rats retain the conditioned place response and express an altered synaptic circuit response to perforant path stimulation of the EC input to DG. This indicates that learning the hippocampusdependent place avoidance is associated with altered neocortical-hippocampal circuit function. The functional alteration of this neural circuit component is specific to the population synaptic component of the response localized to the molecular layer of DG where the stimulated pathway terminates, as observed in both the pre-spike sl-AUC (Fig. 3D) and the sl-fEPSP (Fig. 3E). No change is detected in lacunosum moleculare of CA1 where perforant path fibers also terminate. The change in synaptic circuit function of the entorhinal-to-DG pathway is not observed in no-shock control rats that experienced the identical physical conditions as the conditioned rats with one exception - they were never shocked. Because on average, the conditioned rats only enter the shock zone $\sim 5$ times during each 10-min training session (Fig. 2D), the physical experience of the environment only differs between the conditioned and no-shock groups during shock, which comprised $\sim 2.5 \mathrm{~s}(5 \times 500 \mathrm{~ms})$ or $<1 \%$ of each training session's duration. While the conditioning is sufficient to alter population synaptic function, it also results in increased neuronal discharge, evidenced as an increased PS response at the granule cell layer, whereas the enhanced sl-fEPSP at the granule cell layer is harder to interpret (Fig. $3 F$ ) because the PP terminates at the molecular layer. Within the conditioned DG, the evoked responses are greater (Fig. $3 E-G$ ) and both the pre-spike population synaptic response and the post-spike population response are enhanced compared to the corresponding responses in home-cage DG (Fig. 3D). In contrast, the conditioning does not alter E-S coupling, indicating that synaptic input responses change, likely without an effective change in the corresponding excitability, and the changes are input specific. Some components of the evoked responses are altered in both the conditioned and no-shock groups while other components are altered only in the conditioned group. An enhanced synaptic component of the evoked response was only observed in the conditioned rats, whereas compared to home-cage rats, the measures of neuronal discharge and post-spike activity were enhanced after either the conditioned or non- conditioned experience. Perhaps these changes reflect learning because even the no-shock rats' experience leads to spatial learning as measured by hippocampal physiology (Muller and Kubie, 1987; Wilson and McNaughton, 1993; Radwan et al., 2016).

The observed increases in sl-PS amplitude in the conditioned and no-shock groups compared to the homecage group appears not to be due to increased intrinsic excitability as no significant effect of experience on E-S coupling is observed. However, E-S coupling reflects both intrinsic excitability and the balance of excitation/inhibition (Lu et al., 2000; Daoudal et al., 2002), which cannot be distinguished without additional experiments. Alternatively, either altered feedback or feedforward inhibition could account for the observations, but although the paired-pulse paradigm used in these studies did not detect a group difference, other estimates of feedback inhibition are warranted before concluding that feedback inhibition is unchanged by active place avoidance training. Nonetheless, because learning-induced changes in interneuron connectivity have been described in DG (Ruediger et al., 2011), and inhibition-sensitive increases of Schaffer collateral synapse effectiveness have been observed in conditioned mice (Pavlowsky et al., 2017), it may be the case that learning causes more complex neural circuit changes that might be obscured in the present study by use of anesthesia, which can differentially impact excitatory and inhibitory neurotransmission. The impact of the conditioning and no-shock experiences on feedforward inhibition requires further examination, ideally in awake, freely-behaving subjects.

\section{Relationship to prior work}

Previous work in both awake rats (Whitlock et al., 2006) as well as ex vivo mouse hippocampus slices (Pavlowsky et al., 2017) observed changes in CA3-CA1 synaptic function after avoidance learning, whereas in the mouse slice experiments EC-CA1 synaptic responses were unchanged after place avoidance learning (Pavlowsky et al., 2017), similar to the conditioned rats in the present study. In vivo recordings of anesthetized mouse DG responses to stimulation of EC input showed an increased fEPSP response in trained versus naïve animals while the amplitude of the PS and the E-S coupling was unchanged (Park et al., 2015). While our results are similar to Park et al. (2015), in that we find that the sl-fEPSP is increased with conditioning, our results contrast with these previous results because we also see an impact of conditioning on the sI-PS amplitude. This discrepancy could be due to the differences in analyzing our signals; by recording at multiple, evenly spaced sites, we were able to reduce the influence of volume conduction and better localize our measurements by computing the CSD and making our measurements on the CSD waveforms. Consistent with prior work, we conclude that active place avoidance conditioning causes long-lasting changes to hippocampus circuit function in both mice and rats.

Other forms of conditioning have also been reported to change hippocampus circuit function. Forms of eyeblink conditioning cause LTP-like NMDAR- and PKM $\zeta$-depen- 
dent enhancement of the CA3-CA1 pathway, assessed in awake mice (Gruart et al., 2006; Gruart and DelgadoGarcía, 2007; Madroñal et al., 2010). Non-aversive, nonassociative learning, such as in an object recognition memory task was also associated with enhancement of CA3-CA1 input (Clarke et al., 2010). Changes in EC-DG synaptic responses were found over the course of operant conditioning, as were changes in EC-CA1. The changes were moderate in both cases and were behavior dependent. Remarkably, the EC-DG changes were only measurable when animals approached the lever, and these were only observed during the learning phase of the paradigm and returned to baseline after the task was acquired. Furthermore, changes in EC-CA1 were only significantly altered when animals were going to retrieve food, eating the food, or grooming (Fernández-Lamo et al., 2018). Although it appears that some of these changes are transient (Fernández-Lamo et al., 2018), other changes measured in vivo can persist for days (Park et al., 2015) and cannot be easily explained by a behaviorally-induced, temporary rise in brain temperature (Moser et al., 1993a,b; Moser, 1995). This is especially the case when we consider data from ex vivo slice experiments (Pavlowsky et al., 2017) and under constant conditions of anesthesia (Park et al., 2015), as in the present study. While the present findings unequivocally demonstrate that hippocampus-dependent learning is accompanied by persistent changes to hippocampus circuit function, changes in circuit function may not be unique to hippocampus and may be observable only in specific behavioral states (Fernández-Lamo et al., 2018). It is also not known whether the functional changes indicate LTP and other synaptic changes that, according to the synaptic plasticity and memory hypothesis, underlie long-term memory. Definitive evidence remains elusive (Takeuchi et al., 2014; Ryan et al., 2015; Tonegawa et al., 2015).

\section{Changes in synaptic function and synaptic plasticity}

As described above, we, like others, have observed learning-related group differences in hippocampus circuit function that were detected in vivo by measurements of neural population activity such as the evoked responses of the present study. Because the groups only differ in their experience, we interpret those differences to indicate changes in circuit function consequent to different experiences that are our experimental manipulations. Such changes must be widespread if they are measurable in population activity and are thus at odds with expectations that memory-related functional changes are sparse (Whitlock et al., 2006; Takeuchi et al., 2014). Nonetheless they have been observed, but according to sparsity predictions, they are unlikely to simply reflect the LTP or depression of the specific synapses that store memory.

Here, we observed that some components of the evoked responses are altered in both the conditioned and no-shock groups while other components are altered only in the conditioned group, highlighting that behavioral history is important for interpreting estimates of neural circuit function, and that it can be difficult to definitively attribute measurable changes to specific changes in synaptic func- tion (Moser et al., 1994; Andersen and Moser, 1995; Moser, 1995; Talbot et al., 2018). In particular, we observe altered sl-fEPSP and pre-spike activity in the conditioned group and altered sl-PS and post-spike activity in both the conditioned and no-shock groups. These changes in both the conditioned and no-shock rats could be explained by various mechanisms that have been observed after exposing rodents to enriched environments. In the current study, both the conditioned and no-shock rats were handled as well as exposed to a novel environment, which may serve as enrichment compared to the home-cage rats. The mechanisms by which enrichment induced neuronal alterations include increased neurogenesis (Kempermann et al., 1997, 2002), increased dendritic length (Faherty et al., 2003), increased spine density (Moser et al., 1994), increased synaptic density (Rampon et al., 2000), and increased synaptic protein expression (Frick and Fernandez, 2003; Nithianantharajah et al., 2004) to name a few. Given the impact of enrichment on these mechanisms, it is possible that pre-spike versus postspike alterations are a generalizable feature of conditioning versus experience. While more experiments need to be performed to determine the generality as well as the specific nature of the present observations, the findings nonetheless demonstrate that experience-induced alterations to hippocampus circuit function are widespread but not global; they are instead pathway and compartment specific.

\section{References}

Andersen P, Moser El (1995) Brain temperature and hippocampal function. Hippocampus 5:491-498.

Brankack J, Stewart M, Fox SE (1993) Current source density analysis of the hippocampal theta rhythm: associated sustained potentials and candidate synaptic generators. Brain Res 615:310327.

Clarke JR, Cammarota M, Gruart A, Izquierdo I, Delgado-García JM (2010) Plastic modifications induced by object recognition memory processing. Proc Natl Acad Sci USA 107:2652-2657.

Daoudal G, Hanada Y, Debanne D (2002) Bidirectional plasticity of excitatory postsynaptic potential (EPSP)-spike coupling in CA1 hippocampal pyramidal neurons. Proc Natl Acad Sci USA 99: 14512-14517.

Faherty CJ, Kerley D, Smeyne RJ (2003) A Golgi-Cox morphological analysis of neuronal changes induced by environmental enrichment. Brain Res Dev Brain Res 141:55-61.

Fernández-Lamo I, Delgado-García JM, Gruart A (2018) When and where learning is taking place: multisynaptic changes in strength during different behaviors related to the acquisition of an operant conditioning task by behaving rats. Cereb Cortex 28:1011-1023.

Frick KM, Fernandez SM (2003) Enrichment enhances spatial memory and increases synaptophysin levels in aged female mice. Neurobiol Aging 24:615-626.

Gruart A, Delgado-García JM (2007) Activity-dependent changes of the hippocampal CA3-CA1 synapse during the acquisition of associative learning in conscious mice. Genes Brain Behav 6 [Suppl 1]:24-31.

Gruart A, Muñoz MD, Delgado-García JM (2006) Involvement of the CA3-CA1 synapse in the acquisition of associative learning in behaving mice. J Neurosci 26:1077-1087.

Herreras O (1990) Propagating dendritic action potential mediates synaptic transmission in CA1 pyramidal cells in situ. J Neurophysiol 64:1429-1441. 
Holsheimer J (1987) Electrical conductivity of the hippocampal CA1 layers and application to current-source-density analysis. Exp Brain Res 67:402-410.

Hrabetová S (2005) Extracellular diffusion is fast and isotropic in the stratum radiatum of hippocampal CA1 region in rat brain slices. Hippocampus 15:441-450.

Hsieh C, Tsokas P, Serrano P, Hernández AI, Tian D, Cottrell JE, Shouval HZ, Fenton AA, Sacktor TC (2017) Persistent increased $\mathrm{PKM} \zeta$ in long-term and remote spatial memory. Neurobiol Learn Mem 138:135-144.

Kempermann G, Kuhn HG, Gage FH (1997) More hippocampal neurons in adult mice living in an enriched environment. Nature 386:493-495.

Kempermann G, Gast D, Gage FH (2002) Neuroplasticity in old age: sustained fivefold induction of hippocampal neurogenesis by longterm environmental enrichment. Ann Neurol 52:135-143.

Lu YM, Mansuy IM, Kandel ER, Roder J (2000) Calcineurin-mediated LTD of GABAergic inhibition underlies the increased excitability of CA1 neurons associated with LTP. Neuron 26:197-205.

Madroñal N, Gruart A, Sacktor TC, Delgado-García JM (2010) PKMzeta inhibition reverses learning-induced increases in hippocampal synaptic strength and memory during trace eyeblink conditioning. PLoS One 5:e10400.

Moser El (1995) Learning-related changes in hippocampal field potentials. Behav Brain Res 71:11-18.

Moser E, Mathiesen I, Andersen P (1993a) Association between brain temperature and dentate field potentials in exploring and swimming rats. Science 259:1324-1326.

Moser E, Moser MB, Andersen P (1993b) Synaptic potentiation in the rat dentate gyrus during exploratory learning. Neuroreport 5:317320.

Moser MB, Trommald M, Andersen P (1994) An increase in dendritic spine density on hippocampal CA1 pyramidal cells following spatial learning in adult rats suggests the formation of new synapses. Proc Natl Acad Sci USA 91:12673-12675.

Muller RU, Kubie JL (1987) The effects of changes in the environment on the spatial firing of hippocampal complex-spike cells. J Neurosci 7:1951-1968.

Nithianantharajah J, Levis H, Murphy M (2004) Environmental enrichment results in cortical and subcortical changes in levels of synaptophysin and PSD-95 proteins. Neurobiol Learn Mem 81:200210.

Park EH, Burghardt NS, Dvorak D, Hen R, Fenton AA (2015) Experience-dependent regulation of dentate gyrus excitability by adult-born granule cells. J Neurosci 35:11656-11666.
Pastalkova E, Serrano P, Pinkhasova D, Wallace E, Fenton AA, Sacktor TC (2006) Storage of spatial information by the maintenance mechanism of LTP. Science 313:1141-1144.

Pavlowsky A, Wallace E, Fenton AA, Alarcon JM (2017) Persistent modifications of hippocampal synaptic function during remote spatial memory. Neurobiol Learn Mem 138:182-197.

Radwan B, Dvorak D, Fenton AA (2016) Impaired cognitive discrimination and discoordination of coupled theta-gamma oscillations in Fmr1 knockout mice. Neurobiol Dis 88:125-138.

Rampon C, Tang Y-P, Goodhouse J, Shimizu E, Kyin M, Tsien JZ (2000) Enrichment induces structural changes and recovery from nonspatial memory deficits in CA1 NMDAR1-knockout mice. Nat Neurosci 3:238-244.

Rossetti T, Banerjee S, Kim C, Leubner M, Lamar C, Gupta P, Lee B, Neve R, Lisman J (2017) Memory erasure experiments indicate a critical role of CaMKII in memory storage. Neuron 96:207-216.e2.

Ruediger S, Vittori C, Bednarek E, Genoud C, Strata P, Sacchetti B, Caroni P (2011) Learning-related feedforward inhibitory connectivity growth required for memory precision. Nature 473:514-518.

Ryan TJ, Roy DS, Pignatelli M, Arons A, Tonegawa S (2015) Memory. Engram cells retain memory under retrograde amnesia. Science 348:1007-1013.

Takeuchi T, Duszkiewicz AJ, Morris RGM (2014) The synaptic plasticity and memory hypothesis: encoding, storage and persistence. Philos Trans R Soc Lond B Biol Sci 369:20130288.

Talbot ZN, Sparks FT, Dvorak D, Curran BM, Alarcon JM, Fenton AA (2018) Normal CA1 place fields but discoordinated network discharge in a Fmr1-null mouse model of fragile $X$ syndrome. Neuron 97:684-697.e4.

Tonegawa S, Pignatelli M, Roy DS, Ryan TJ (2015) Memory engram storage and retrieval. Curr Opin Neurobiol 35:101-109.

Tsokas $\mathrm{P}$, Hsieh $\mathrm{C}$, Yao $\mathrm{Y}$, Lesburguères $\mathrm{E}$, Wallace EJC, Tcherepanov A, Jothianandan D, Hartley BR, Pan L, Rivard B, Farese RV, Sajan MP, Bergold PJ, Hernández Al, Cottrell JE, Shouval HZ, Fenton AA, Sacktor TC (2016) Compensation for PKM $\zeta$ in longterm potentiation and spatial long-term memory in mutant mice. Elife 5:e14846.

Whitlock JR, Heynen AJ, Shuler MG, Bear MF (2006) Learning induces long-term potentiation in the hippocampus. Science 313: 1093-1097.

Wilson MA, McNaughton BL (1993) Dynamics of the hippocampal ensemble code for space. Science 261:1055-1058.

Wu K, Leung LS (2003) Increased dendritic excitability in hippocampal ca1 in vivo in the kainic acid model of temporal lobe epilepsy: a study using current source density analysis. Neuroscience 116: 599-616. 